\title{
Addition of dimethylsulphoxide to methyl-tert-butyl ether and ethyl propionate increases cholesterol dissolving capacity and cholesterol gall stone dissolution in vitro
}

\author{
J J G H M Bergman, A K Groen, K Huibregtse, G N J Tytgat
}

\begin{abstract}
There is a discrepancy between in vitro cholesterol dissolving efficacy of methyltert-butyl ether (MTBE) and ethyl propionate and cholesterol gall stone dissolution in vivo. This study investigated whether the presence of bile changes the cholesterol dissolving capacity of MTBE and ethyl propionate. The addition of dimethylsulphoxide to MTBE or ethyl propionate was also studied to discover if it improves the dissolving capacity for cholesterol gall stones. The presence of bile caused a $25 \%$ decrease in cholesterol dissolving capacity of both MTBE and ethyl propionate $(p<0.0001)$. This inhibitory effect of bile could be overcome by the addition of dimethylsulphoxide: dimethylsulphoxide caused an increase in cholesterol dissolving capacity of MTBE and ethyl propionate, the increase depending on the dimethylsulphoxide/bile ratio in the mixture. Mean dissolution time of weight, size, and patient matched cholesterol gall stones was 220 minutes in MTBE and 130 minutes in MTBE/dimethylsulphoxide $(p<0 \cdot 0001)$. No stones dissolved completely in ethyl propionate or ethyl propionate/dimethylsulphoxide within 300 minutes. In conclusion, MTBE/dimethylsulphoxide is a more potent dissolving agent for cholesterol gall stones than MTBE, giving a $40 \%$ reduction in dissolution time. Addition of dimethylsulphoxide to ethyl propionate does not result in faster stone dissolution. MTBE and MTBE/dimethylsulphoxide are far superior to ethyl propionate as solvents for cholesterol gall stones.

(Gut 1994; 35: 1653-1658)
\end{abstract}

Department of

Gastroenterology of

the Academic Medical

Centre of the

University of

Amsterdam, The

Netherlands

J J G H M Bergman

A K Groen

K Huibregtse

G N J Tytgat

Correspondence to:

Dr J J G H M Bergman,

Academic Medical Centre, Department of

Gastroenterology,

Meibergdreef $9,1105 \mathrm{AZ}$

Amsterdam, The

Netherlands.

Accepted for publication 22 February 1994
Contact dissolution treatment of gall bladder stones, using methyl-tert-butyl ether (MTBE) or ethyl propionate, is successful in about $95 \%$ of selected cases. ${ }^{1-4}$ MTBE and to a lesser extent ethyl propionate have proved to be effective solvents for cholesterol in vitro. ${ }^{5}$ There is, however, a clear discrepancy between in vitro efficacy of MTBE and ethyl propionate as cholesterol solvents and time required for dissolution of gall stones in vivo. This is one of the important drawbacks of contact dissolution treatment: treatment is labour intensive and time consuming, negatively influencing cost efficacy. Although studies have been published describing an automated pump, such a device has not become commercially available since publication seven years ago. ${ }^{6-8}$ Treatment therefore still requires manual flushing of the gall bladder for 4-16 hours during several days. ${ }^{12}$ Changes to the treatment regimen resulting in faster stone dissolution can thus be of major importance.

Two factors are known to inhibit dissolution of stones in vivo. Smith et al showed that gallstone dissolution in vitro was inhibited by insoluble stone matrix, existing of calcium, bilirubin, and protein. ${ }^{9} 10$ They speculated that the matrix limits stone dissolution because of its spatial distribution within the stones as concentric rings that retard contact of the cholesterol solvent with underlying layers of crystalline cholesterol. Addition of a matrix dissolving agent has been shown to accelerate dissolution of gall stones in vitro. ${ }^{11-13}$

Less attention has been given to stonesolvent contact as a factor influencing contact dissolution in vivo: MTBE/bile and ethyl propionate/bile are biphasic mixtures with a lighter ether phase above and a heavier bile phase below. Because gall stones have a higher density than bile, they sink into the bile phase and do not come in close contact with the dissolving agents in the upper phase. ${ }^{14-16}$ Creating turbulence manually as during treatment in vivo can only partially overcome this effect.

So far the effect of bile components on cholesterol dissolution in MTBE and ethyl propionate has not been investigated. Almost all in vitro studies on solvents for gall stones have been performed in the absence of bile. Therefore such an effect cannot be excluded.

Addition of dimethylsulphoxide to MTBE or ethyl propionate could enhance stone dissolution in vivo. Dimethylsulphoxide is a potent solvent for bilirubin and easily mixes with MTBE and ethyl propionate in contrast with other stone matrix dissolving agents like EDTA or $N$-acetylcysteine. ${ }^{17} \mathrm{By}$ its dissolving effect on bilirubin dimethylsulphoxide could overcome part of the shielding effect of the stone matrix on cholesterol dissolution in MTBE or ethyl propionate. Dimethylsulphoxide could also improve stone solvent contact. ${ }^{16}$ In the presence of bile, monophasic mixtures of MTBE/dimethylsulphoxide or ethyl propionate/dimethylsulphoxide become biphasic: MTBE or ethyl propionate in the upper phase, dimethylsulphoxide/bile in the 
lower phase. This probably reflects the high affinity of the bipolar dimethylsulphoxide for aqueous media and the poor solubility of bile in MTBE. Because dimethylsulphoxide has a higher density than bile, the density of the lower phase increases. Stones that sink into the bile phase will float at the interphase, coming in closer contact with the dissolving agents of the upper phase. Presence of dimethylsulphoxide could change the cholesterol solubilising capacity of MTBE and ethyl propionate in a similar way, however, as bile might have a negative effect on cholesterol dissolution in these solvents.

The aims of this study were twofold. Firstly, to test if the presence of bile changes the cholesterol dissolving capacity of MTBE and ethyl propionate, and secondly to investigate if addition of dimethylsulphoxide improves the dissolution capacity of MTBE and ethyl propionate for cholesterol gall stones.

\section{Methods}

SOLVENTS AND BILE

MTBE, ethyl propionate, and dimethylsulphoxide (all synthetical grade) were purchased from Merck (Darmstadt, Germany). Cholesterol (monohydrate, 99\% pure) was provided by Sigma Chemical Company (St Louis, MO). Hepatic bile was collected from one single patient by a $\mathrm{T}$ drain after cholecystectomy. To eliminate changes in bile composition because of surgery, bile collection was started three days postoperatively.

GALL STONES

Human cholesterol gall stones were obtained at laparoscopic cholecystectomy. Gall stones were selected from patients whose gall bladders contained more than 10 stones similar in size and morphology. Black pigment stones were identified visually and excluded from this study. A total of 46 gall stones obtained from three patients were selected. Stones from each patient were kept separate from those of other patients and stored in bile at $-20^{\circ} \mathrm{C}$. To verify if stones were actually cholesterol gall stones one stone from each patient was subjected to compositional analysis. All stones were incubated in bile and $x$ rayed using the same settings as for plain abdominal $x$ ray. Only stones that were completely radiolucent were used for dissolution experiments. After washing in deionised distilled water to remove bile and debris, stones were air dried for 30 minutes. Using a Mettler HL52 balance, individual gall stone weight was determined. Maximum and minimum diameter were measured using calipers and rulers. For paired dissolution experiments 13 stone pairs, matched for weight, size, and patient, were selected. A difference of more than $10 \%$ between two stones of the same stone pair was not permitted in respect to weight or sum of maximal and minimal diameter.
INFLUENCE OF BILE AND WATER ON CHOLESTEROL DISSOLUTION CAPACITY OF MTBE AND ETHYL PROPIONATE

Mixtures of MTBE/bile, MTBE/water, ethyl propionate/bile, and ethyl propionate/water were made in volume ratios of $90 / 10,80 / 20$, $70 / 30$, and $50 / 50$ such that total volume of each mixture was $5 \mathrm{ml}$. All mixtures were biphasic: the lighter MTBE or ethyl propionate phase above, the heavier bile or water phase below. To each biphasic mixture an excess of pure cholesterol crystals was added

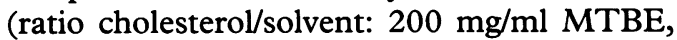
$100 \mathrm{mg} / \mathrm{ml}$ ethyl propionate). After addition of cholesterol, mixtures were incubated at room temperature in a REAX 2 circulator $(60 \mathrm{rpm})$ for 30 minutes. The mixtures were then centrifuged at $2000 \mathrm{rpm}$ for two minutes and cholesterol concentration in the upper phase (MTBE or ethyl propionate) was determined using the cholesterol oxidase method (Boeringer, Mannheim). The cholesterol concentration measured was considered to represent the maximum cholesterol dissolving capacity of the solvent at room temperature.

\section{EFFECT OF ADDITION OF}

DIMETHYLSULPHOXIDE TO MTBE/BILE AND

ETHYL PROPIONATE/BILE MIXTURES ON

CHOLESTEROL DISSOLUTION CAPACITY OF

MTBE AND ETHYL PROPIONATE

Monophasic MTBE/dimethylsulphoxide and ethyl propionate/dimethylsulphoxide mixtures were prepared in volume ratios of $90 / 10$, $80 / 20,70 / 30$, and $50 / 50$. These mixtures were then added to different amounts of bile to give final percentages of $10,20,30$, and $50 \%$ of bile. The total volume of each mixture was $5 \mathrm{ml}$. Addition of bile caused an immediate phase separation leading to biphasic mixtures with an upper MTBE or ethyl propionate phase and a lower phase consisting of dimethylsulphoxide and bile. This phase separation was an exothermic process causing a rise in temperature of about $8^{\circ} \mathrm{C}$ in every mixture. To ensure that dissolution tests were performed under isothermic conditions all MTBE/dimethylsulphoxide/bile and ethyl propionate/dimethylsulphoxide/bile mixtures were circulated in a REAX 2 circulator $(60 \mathrm{rpm})$ for 30 minutes before addition to cholesterol. To each mixture an excess of cholesterol was added (ratio cholesterol/solvent: $200 \mathrm{mg} / \mathrm{ml}$ MTBE, $100 \mathrm{mg} / \mathrm{ml}$ ethyl propionate). Mixtures were subsequently treated as described above. During the dissolution experiments no change of temperature was seen.

\section{PAIRED STONE DISSOLUTION EXPERIMENTS}

Of the 13 stone pairs selected, seven were used to compare stone dissolution in MTBE with a dissolution regimen using $\mathrm{MTBE} /$ dimethylsulphoxide $(70 / 30)$. The other six stone pairs were subjected to dissolution in ethyl propionate and ethyl propionate/dimethylsulphoxide $(70 / 30)$. Stones were put in separate glass test tubes in which the tip of a polyethylene 


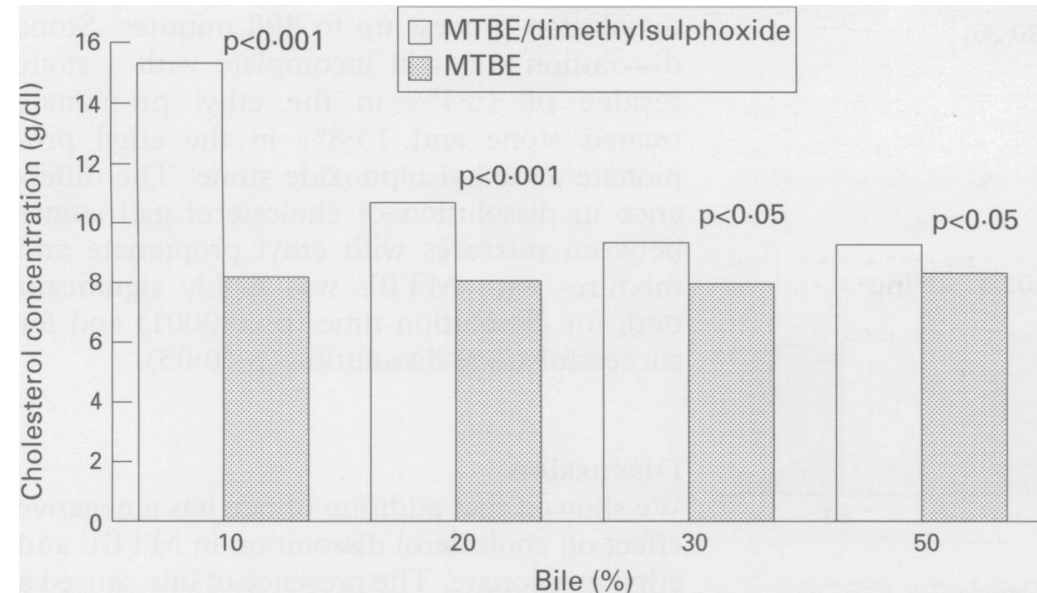

Figure 1: Cholesterol concentration in MTBE phases of MTBE/dimethylsulphoxide $(80 / 20) /$ bile and MTBE/bile mixtures after addition of an excess of cholesterol.

nasobiliary pigtail catheter (diameter $7 \mathrm{~F}$, length $120 \mathrm{~cm}$ ), was placed. This same type of catheter is used in transpapillary contact dissolution treatment in vivo in our unit. Hepatic bile $(2 \mathrm{ml})$ was added and gall stones were subsequently dissolved in $8 \mathrm{ml}$ of either MTBE, MTBE/dimethylsulphoxide (70/30), ethyl propionate or ethyl propionate/dimethylsulphoxide (70/30). The in vivo situation was mimicked by creating turbulence manually using glass syringes (4-6 cycles a minute) and by exchanging solvents and bile every 15 minutes. Test tubes were incubated in the dark at $37^{\circ} \mathrm{C}$. Every 15 minutes gall stones were air dried for three minutes and weighed on a Mettler HL52 balance. Dissolution was expressed as the percentage of initial dry stone weight remaining at each time point. If during the dissolution process stones disintegrated no further weight determination was performed until the end of the experiment to avoid artificial fragmentation caused by handling of the fragments. The experiment was stopped whenever gall stones had completely dissolved or a time limit of 300 minutes had passed.

\section{STATISTICS}

Results are expressed as percentages or mean

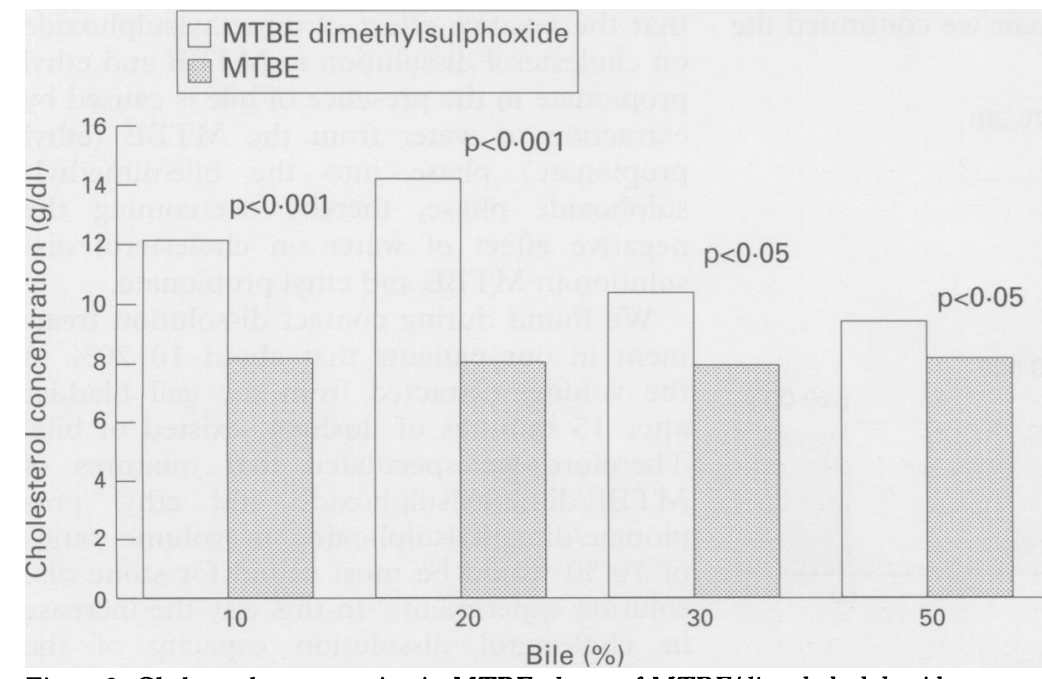

Figure 2: Cholesterol concentration in MTBE phases of MTBE/dimethylsulphoxide (70/30)/bile and MTBE/bile mixtures after addition of an excess of cholesterol.
(SD). Data were compared using (paired) Student's $t$ test.

\section{Results}

INFLUENCE OF WATER AND BILE ON CHOLESTEROL DISSOLUTION CAPACITY OF MTBE AND ETHYL PROPIONATE

Compared with pure MTBE or ethyl propionate, the presence of water or bile caused a $25 \%$ decrease in cholesterol dissolution capacity of both solvents. In MTBE decrease in cholesterol dissolution capacity was $25 \%$ (95\% confidence intervals: 22 to $27 \%, \mathrm{p}<0.00001$ ). In ethyl propionate mean decrease in cholesterol concentration was $28 \%$ (95\% confidence intervals: 23 to $34 \%, \mathrm{p}<0.00001)$. Data are given as mean of four separate experiments. Standard deviation were less than $5 \%$. For this negative effect on cholesterol dissolution only small amounts $(5 \%)$ of water or bile were necessary. Increasing the volume ratio of water or bile in the mixtures beyond this $5 \%$ did not cause any further decrease in cholesterol dissolution capacity of the solvents. There was no difference in effect on cholesterol dissolution between addition of water or bile.

\section{EFFECT OF ADDITION OF}

DIMETHYLSULPHOXIDE TO MTBE/BILE AND ETHYL PROPIONATE/BILE MIXTURES ON CHOLESTEROL DISSOLUTION CAPACITY OF MTBE AND ETHYL PROPIONATE

Figures 1 and 2 show the mean cholesterol concentration in MTBE phases of MTBE/ dimethylsulphoxide/bile and MTBE/bile mixtures after addition of an excess of cholesterol. Figures 3 and 4 show this information for ethyl propionate/dimethylsulphoxide/bile and ethyl propionate/bile mixtures. Data are given as mean of four separate experiments. Standard deviation were less than $5 \%$. In mixtures containing dimethylsulphoxide, the MTBE and ethyl propionate phases had a significantly higher mean cholesterol concentration than in the mixtures without any dimethylsulphoxide, $p<0.001$. The increase in cholesterol dissolution capacity depended on the percentage of bile in the mixtures, as shown in Figures 1-4 separately.

The differences between Figures 1 and 2 and Figures 3 and 4 show, however, that changing the MTBE/dimethylsulphoxide or ethyl propionate/dimethylsulphoxide ratio, also changed the cholesterol dissolution capacity of the solvents. Therefore the dimethylsulphoxide/bile ratio was calculated for all mixtures and related to the cholesterol concentration in the MTBE or ethyl propionate phases (Fig 5). Increase in cholesterol dissolution capacity clearly depended on the dimethylsulphoxide/bile ratio in the mixture, a rise of about $100 \%$ being reached at a ratio of about 2 . Both MTBE and ethyl propionate showed the same effect, however cholesterol dissolution capacity of ethyl propionate was about $50 \%$ of that of MTBE. 


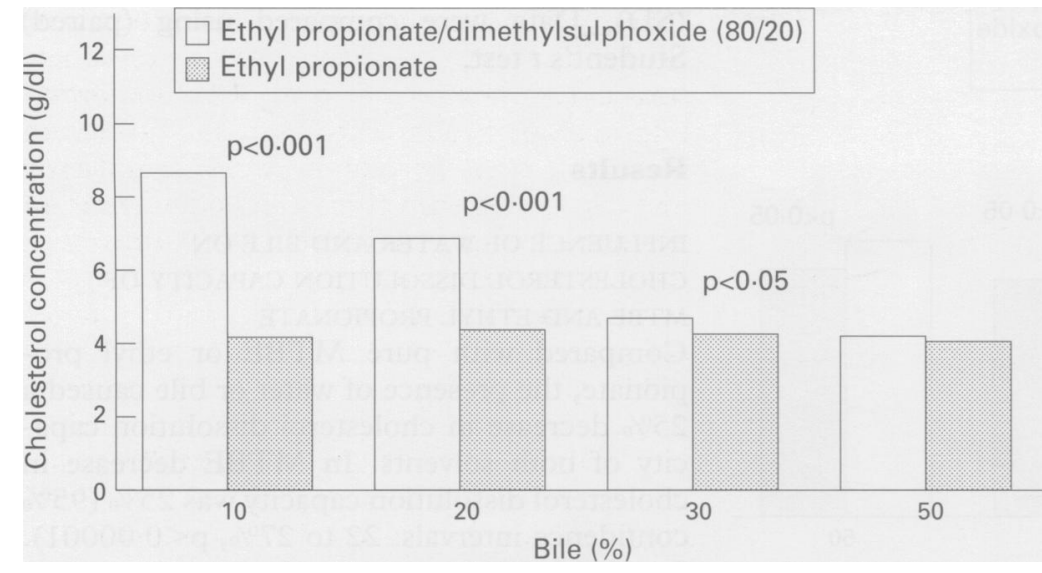

Figure 3: Cholesterol concentration in ethyl propionate phases of ethyl propionate/dimethylsulphoxide (80/20)/bile and ethyl propionate/bile mixtures after addition of an excess of cholesterol.

PAIRED STONE DISSOLUTION TESTS

All stones used in paired dissolution experiments were radiolucent in $x$ ray and had a cholesterol content higher than $95 \%$. Stones added to MTBE or ethyl propionate all sank into the bile phase whereas stones treated with MTBE/dimethylsulphoxide or ethyl propionate/dimethylsulphoxide all floated at the interphase. This difference in stone solvent contact, however, was probably minimised by the turbulence created manually with glass syringes. In MTBE and MTBE/dimethylsulphoxide mixtures all cholesterol gall stones dissolved completely. Mean time required for complete stone dissolution was 220 minutes in MTBE (SD 21 minutes, range 180-240 minutes) and 130 minutes in MTBE/dimethylsulphoxide (SD 28 minutes, range 105-180). Figure 6 shows the results of these paired dissolution tests. Compared with MTBE, MTBE/dimethylsulphoxide mixtures gave a faster stone dissolution in every stone pair. Mean time reduction was 90 minutes (95\% confidence intervals: 65 to 115 minutes, $\mathrm{p}<0.0001$ ).

In ethyl propionate and ethyl propionate/dimethylsulphoxide no cholesterol gall stone dissolved completely within the time frame of 300 minutes, leaving a mean stone residue of $35 \%$ in ethyl propionate and $28 \%$ in ethyl propionate/dimethylsulphoxide mixtures $(p=0 \cdot 13)$. In one stone pair we continued the

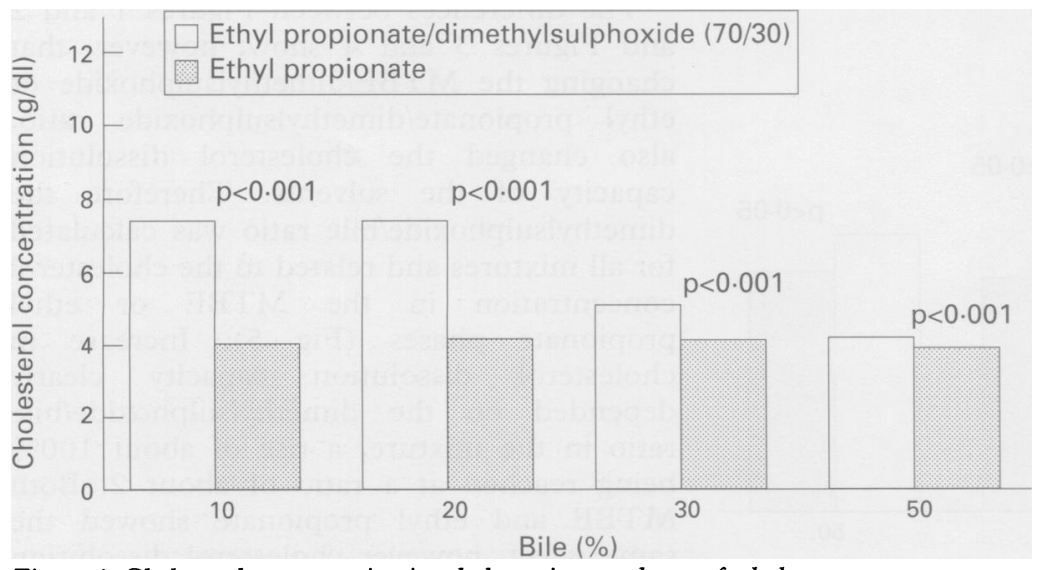

Figure 4: Cholesterol concentration in ethyl propionate phases of ethyl propionate/dimethylsulphoxide (70/30)/bile and ethyl propionate/bile mixtures after addition of an excess of cholesterol. dissolution process up to 480 minutes. Stone dissolution was still incomplete with a stone residue of $16.7 \%$ in the ethyl propionate treated stone and $15.8 \%$ in the ethyl propionate/dimethylsulphoxide stone. The difference in dissolution of cholesterol gall stones between mixtures with ethyl propionate and mixtures with MTBE was highly significant both for dissolution time $(p<0.0001)$ and for successful stone dissolution $(\mathrm{p}<0 \cdot 05)$.

\section{Discussion}

We showed that addition of bile has a negative effect on cholesterol dissolution in MTBE and ethyl propionate. The presence of bile caused a $25 \%$ decrease in the cholesterol dissolving capacity of both MTBE and ethyl propionate. There was no difference between addition of water or bile, suggesting that the inhibitory effect of bile on cholesterol dissolution in MTBE and ethyl propionate is probably caused by its water component. The fact that only small amounts of water or bile were necessary to cause this effect reflects in our opinion the poor solubility of water in MTBE and ethyl propionate. Only a small amount of water is sufficient to completely saturate the solvent with water and to cause the decrease in cholesterol dissolving capacity. Addition of more water does not result in a higher concentration of water in the ether phase and thereby has no further effect on cholesterol dissolution. Because of the negative effect of water and bile on cholesterol dissolution in MTBE and ethyl propionate, we feel that all in vitro testing on solvents for contact dissolution of gall stones should be performed in the presence of water or bile.

The negative effect of bile can be compensated by the addition of dimethylsulphoxide: in our experiments we showed that addition of dimethylsulphoxide to MTBE/bile or ethyl propionate/bile mixtures increases the cholesterol dissolving capacity of MTBE and ethyl propionate compared with mixtures without dimethylsulphoxide. This increase depends on the dimethylsulphoxide/water ratio of the mixture, a maximum increase of $100 \%$ being reached at a ratio of 2 . We hypothesise that the positive effect of dimethylsulphoxide on cholesterol dissolution in MTBE and ethyl propionate in the presence of bile is caused by extraction of water from the MTBE (ethyl propionate) phase into the bile/dimethylsulphoxide phase, thereby overcoming the negative effect of water on cholesterol dissolution in MTBE and ethyl propionate.

We found during contact dissolution treatment in our patients that about $10-20 \%$ in the volume extracted from the gall bladder after 15 minutes of flushing, existed of bile. Therefore we speculated that mixtures of MTBE/dimethylsulphoxide and ethyl propionate/dimethylsulphoxide in volume ratios of $70 / 30$ would be most suited for stone dissolution experiments. In this way the increase in cholesterol dissolution capacity of the solvents could compensate for the loss of absolute volume of solvent entered in the gall 


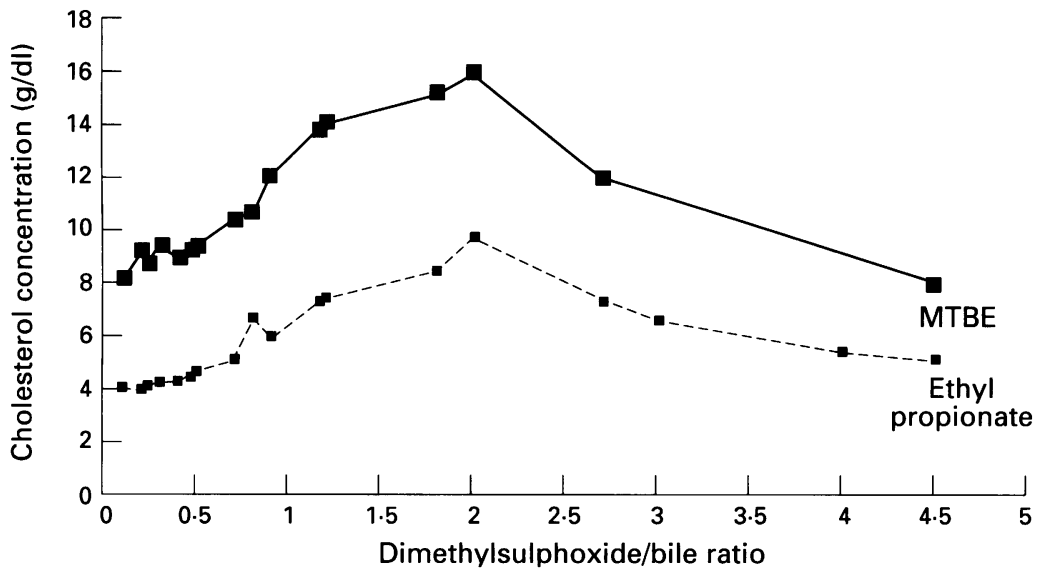

Figure 5: Relation between dimethylsulphoxide/bile ratio and cholesterol concentration of $M T B E$ and ethyl propionate phases in MTBE/dimethylsulphoxide/bile and ethyl propionate/dimethylsulphoxide/bile mixtures.

bladder at every cycle. More importantly, the volume of dimethylsulphoxide entered in the gall bladder would be enough to raise the density of the bile phase and to exert its dissolving effect on the stone matrix.

Our paired stone dissolution tests show that addition of dimethylsulphoxide to MTBE results in a $40 \%$ time reduction compared with a treatment regimen with only MTBE. This time reduction achieved by addition of dimethylsulphoxide is impressive, a mean reduction of 90 minutes on a total dissolution time of 220 minutes. The time required for complete stone dissolution in MTBE is in agreement with in vivo data from other studies in which solitary stones were dissolved after a mean dissolution time of four hours. ${ }^{2}$ We feel that this supports the adequacy of our in vitro model for gall stone dissolution.

In ethyl propionate mixtures addition of dimethylsulphoxide does not result in a significant change in dissolution time. We speculate that the more polar molecular structure of ethyl propionate causes ethyl propionate to compete with bilirubin for dissolution in dimethylsulphoxide.

Stone dissolution experiments were performed in pairs. The main analysis was aimed at comparing MTBE with MTBE/dimethylsulphoxide and ethyl propionate with ethyl propionate/dimethyl-

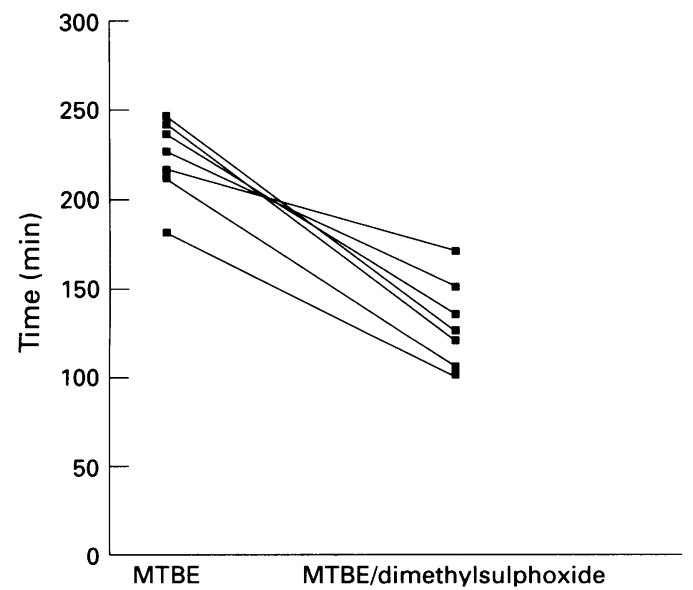

Figure 6: Time required for complete dissolution of pairs of matched cholesterol gall stones dissolved in either MTBE or MTBE/dimethylsulphoxide in the presence of bile. sulphoxide. Comparing mean dissolution time of MTBE mixtures and ethyl propionate mixtures, however, provides important information. Stone pairs used in dissolution tests with ethyl propionate were significantly lower in weight than stone pairs obtained from the same patient dissolved in mixtures with MTBE. Any bias resulting from stone size or weight was therefore directed towards a faster stone dissolution in ethyl propionate.

MTBE mixtures were far superior to ethyl propionate mixtures both for dissolution time and ultimate successful dissolution. We think that these results reflect the higher cholesterol dissolving capacity of MTBE (about twice that of ethyl propionate) and its lower viscosity. This contrasts with the results of Zakko et al who found ethyl propionate more effective in dissolving gall stones in vitro than $\mathrm{MTBE} .{ }^{18}$ In their study they used a more artificial model with an automated high flow system. Because such a pump is not available commercially and has not been approved by the Food and Drug Administration we feel that our system represents the in vivo situation in a more proper way.

After MTBE dissolution insoluble debris may remain. ${ }^{12}$ This might act as a nidus for gall stone recurrence. Nelson et al found this debris to exist predominantly of calcium bilirubin and calcium carbonate. ${ }^{19}$ They showed that a mixture of EDTA with either cholate or polysorbate was most efficient in dissolving these components. Dimethylsulphoxide also has dissolving capacity for these substances and can, in contrast with hydrophilic stone matrix solvents like EDTA or $N$-acetylcysteine, be mixed with MTBE, making application in vivo more favourable. ${ }^{17} 19$ Addition of dimethylsulphoxide to MTBE could thus not only result in faster stone dissolution but could also lead to less residual debris after dissolution. In our studies residual debris after successful dissolution with MTBE or MTBE/dimethylsulphoxide was too small to show such a difference. Others have, however, shown this effect using different matrix solvents. ${ }^{111220}$

Dai et al studied the cytotoxicity of MTBE and MTBE/dimethylsulphoxide (70/30) infused in gall bladders of rabbits. ${ }^{16}$ Their histological study of several rabbit tissues showed that chemical irritation by MTBE/dimethylsulphoxide (70/30) was identical to that caused by MTBE $(100 \%)$ and localised to the level of the gall bladder wall. Normal morphology was rapidly recovered in two weeks and no changes were seen in liver cells, duodenum, kidney or medulla. Somnolence occurred more frequently in the MTBE group suggesting that systemical absorption of MTBE was not increased by the presence of dimethylsulphoxide.

We conclude that using MTBE/dimethylsulphoxide (70/30) instead of MTBE may result in a considerable gain of time in contact dissolution treatment in vivo. Compared with ethyl propionate, MTBE is a superior solvent for contact dissolution in vitro. Addition of dimethylsulphoxide to MTBE may also result 
in a more complete stone dissolution, leaving less residual debris. Toxicity of MTBE/ dimethylsulphoxide seems to be comparable with MTBE. Further testing, however, especially of toxicity in humans is mandatory before application in vivo.

The results of these studies were presented in part at the annua meeting of the American Gastroenterological Association in May 1993 in Boston and were published in abstract form in Gastroenterology 1993; 104: A350.

This work was supported by the Dutch National Health Insurance Board (grant: OG 93/051).

1 Thistle JL, May GR, Bender CE, Williams HJ, LeRoy AJ, Nelson PE, et al. Dissolution of cholesterol gallbladde stones by methyl tert butyl ether administered by a percutaneous transhepatic catheter. N Engl f Med 1989; 320 633-9.

2 Leuschner $U$, Hellstern A, Schmidt $K$, Fisher $H$, Güldütuna $\mathrm{S}$, Hubner $\mathrm{K}$, et al. Gallstone dissolution with methyl tert butyl ether in 120 patients: efficacy and safety. Dig Dis Sci 1991; 36: 193-9.

3 Esch O, VanSonnenberg E, O'Laoide R, Schteingart CD, Wahrenbrock E, Kirby S, et al. Successful contact dissolution of cholesterol gallstones in a high risk patient with ethyl propionate (EP). Gastroenterology 1992; 102: A311.

Esch O, Schteingart CD, Pappert D, Kirby D, Streich R Hofmann AF. Increased blood levels of methyl tert buty ether but not of ethyl propionate during instillation with contact dissolution agents in the pig. Hepatology 1993; 18: 373-9.

5 Flynn GL, Shah Y, Prakongpan S, Kwan KH, Higuchi WI, Hofmann AF. Cholesterol solubility in organic solvents. 7 Pharm Sci 1979; 68: 1090-7.

6 Zakko SF, Hofmann AF, Schteingart C, van Sonnenberg L, Wheeler O. Percutaneous gallbladder stone dissolution using a microprocessor assisted solvent transfer (MAST) using a microprocessor assisted solvent tran

7 Zakko SF, Hofmann AF. Microprocessor assisted solvent transfer system for gallstone dissolution. In vitro and in vivo validation. Gastroenterology 1990; 99: 1807-13.
8 Zakko SF, Hofmann AF. Microprocessor assisted solvent transfer system for effective contact dissolution of gallbladder stones. IEFE Trans Biomed Eng 1990; 37: 410-6. 9 Smith BF. Effect of laser fragmentation of cholesterol and mixed cholesterol gallstones on in vitro dissolution in methyl tert butyl ether. Dig Dis Sci 1990; 35: 236-41.

10 Smith BF, LaMont JT. Identification of gallbladder mucinbilirubin complex in human cholesterol gallstone matrix $f$ Clin Invest 1985; 76: 439-45.

$11 \mathrm{Niu} N$, Smith BF. Addition of $\mathrm{N}$-acetylcysteine to aqueous model bile systems accelerates dissolution of cholesterol gallstones. Gastroenterology 1990; 98: 454-63.

12 Lesma A, McCullough JE, Thistle JL. Mucolytic agent enhance methyl tert butyl ether dissolution of cholesterol enhance methyl tert butyl ether dissolution of choleste

13 Wosiewitz U, Güldütuna S, Fischer H, Leuschner U. Pig ment gallstone dissolution in vitro. Solubilization of brown bilirubinate and black polybilirubinate stone material by buffered solvents, containing EDTA, bile salts, and reducing thiols. Scand f Gastroenrerol 1989; 24: 373-80.

14 Allen MJ, Borody TJ, Thistle JL. In vitro dissolution of cholesterol gallstones. Gastroenterology 1985; 89: 1097-103.

15 Zhou J, Lee SH, Rawat B, Fache JS, Maciejewska U, Burhenne HJ. Iodinated contrast medium as an aid to gallstone dissolution with methyl tert butyl ether: in vitro gallstone dissolution with methyl tert

16 Dai KY, Montet JC, Zhao XM, Amic J, Choux R. Dissolving agents of human mixed cholesterol stones. Gastroenterol Clin Biol 1988; 12. 312-9.

17 Dai KY, Montet JC, Zhao XM, Amic J, Montet AM Dissolution of human brown pigment biliary stones. f Hepatol 1989; 9: 301-11.

18 Zakko SF, Scirica JC, Guttermuth CF, Dodge J, Rashid S, Kahn S, et al. Ethyl propionate (EP) for cholesterol gallstone (GS) dissolution: more effective and less cytotoxic than MTBE. Gastroenterology 1992; 102: A339.

19 Nelson PE, Moyer TP, Thistle JL. Dissolution of calcium bilirubinate and calcium carbonate debris remaining after methyl tert butyl ether dissolution of cholesterol gallmethyl tert butyl ether dissolution of ch

20 Wosiewitz U, Sabinski F, Haus Ch, Güldütuna $S$ Leuschner U. Experimental dissolution of pigment gallstone material using alkaline EDTA and adjuvant bile salts/non-bile salt detergents, thiols and urea, with respect to local chemolitholysis. $\mathcal{F}$ Hepatol 1992; 14: 7-15. 